\title{
A Unique Wavelet-based Multicarrier System with and without MIMO over Multipath Channels with AWGN
}

\author{
Rameez Asif \\ MIEEE, MIET \\ University of Bradford \\ United Kingdom
}

\author{
Raed Abd-Alhameed \\ FIEEE, FIET \\ University of Bradford \\ United Kingdom
}

\author{
J. M. Noras \\ University of Bradford \\ United Kingdom
}

\begin{abstract}
Recent studies suggest that multicarrier systems using wavelets outperform conventional OFDM systems using the FFT, in that they have well-contained side lobes, improved spectral efficiency and BER performance, and they do not require a cyclic prefix. Here we study the wavelet packet and discrete wavelet transforms, comparing the BER performance of wavelet transform-based multicarrier systems and Fourier based OFDM systems, for multipath Rayleigh channels with AWGN. In the proposed system zero-forcing channel estimation in the frequency domain has been used. Results confirm that discrete wavelet-based systems using Daubechies wavelets outperform both wavelet packet transform- based systems and FFT-OFDM systems in terms of BER. Finally, Alamouti coding and maximal ratio combining schemes were employed in MIMO environments, where results show that the effects of multipath fading were greatly reduced by the antenna diversity.
\end{abstract}

\section{Keywords}

OFDM, BER, Multipath, Discrete Wavelet Transform (DWT), Wavelet Packet Transform (WPT), Multiple Input Multiple Output (MIMO)..

\section{INTRODUCTION}

Contemporary communication systems employ a number of different techniques for signal transmission, reception and processing [1]. High level methodologies such as multiplexing in frequency [2], [3], in time [4] and spatially [5], which are now in common use, are undergoing extensive research in the search for highest quality of service.

Orthogonal Frequency Division Multiplexing (OFDM) is the main contender for the communication systems of the next generation. In this multicarrier modulation technique, the total channel bandwidth is divided into different sub-bands and the data symbols are then transmitted over these sub-bands. This division of the channel bandwidth provides high tolerance against multipath fading [6]. OFDM is now accepted as a standard in asymmetric digital subscriber line (ADSL) and digital video broadcasting (DVB), and in WiMAX and IEEE 802.11 [7].

However OFDM does have major disadvantages, namely strong side lobes, high peak to average power ratio (PAPR), and sensitivity to carrier timing offset [8].

In consideration of the above OFDM limitations, recent studies have suggested wavelet-based multicarrier systems [9]-[12] as an alternative to conventional FFT-OFDM, using the Discrete Wavelet Transform (DWT), or the Wavelet Packet Transform (WPT). This may provide all the advantages of conventional OFDM, but also mitigate the problems of PAPR and carrier timing offset [13]-[15].

In fast Fourier analysis a signal is decomposed into its constituent individual sinusoids, whereas a wavelet transform decomposes signal into different bands of the frequency spectrum, with the same computational complexity of order $\mathrm{O}\left(\mathrm{N} \log _{2} \mathrm{~N}\right)$ [16] for discrete cosine while many other types of wavelet transforms in which FFT is approximated using wavelets can be effectively computed with the time complexity of $\mathrm{O}(\mathrm{N})$ [17]. Inter-symbol interference (ISI) and inter-carrier interference (ICI) arising from the loss of orthogonality of transmitted symbols in OFDM can be countered by the use of wavelets and in [18], the effect of narrowband interference has been shown to be more pronounced in OFDM using the FFT rather than the DWT.

The wavelet-based system achieves orthogonality through the use of orthogonal wavelet filters, also referred to as filter banks [19]. The DWT produces narrow side lobes with large power spectral density. No cyclic prefix insertion is required, which can save up to $25 \%$ of the bandwidth making waveletbased multicarrier systems more bandwidth efficient [20]-[24] and enabling improved BER performance. The wavelet transform represents signals jointly in the time and frequency domains, using multi-resolution analysis. This property of wavelets also makes them suitable for treating signals with exotic spectral properties, for example signals that have timedependent spectral properties.

More recently, the present authors in R. Asif et al [25] have proposed DWT for multi-carrier modulations using time domain equalization. In this study we present a new way of channel equalization when working with wavelets. We examine the ISI, and compare the performance of different wavelet families in terms of signal reconstruction. DWT and WPT methods are studied in terms of processing time and performance. Another major contribution of this study is the extension of the DWT technique into the MIMO environment where the performance of the system has been evaluated using both receive and transmit diversity techniques.

Section II of this paper illustrates the baseband architecture and implementation of the proposed system. The simulation results are discussed in Section III and Section IV contains the conclusions.

\section{DWT BASEBAND ARCHITECTURE AND IMPLEMENTATION}

The continuous-time wavelet transform (CWT) can be written [26]:

$$
\begin{aligned}
\operatorname{CWTx}(\tau, a)=\left\langle x(t), \psi_{a, \tau}\right\rangle \\
\quad=\frac{1}{\sqrt{|a|}} \int_{-\infty}^{\infty} x(t) \psi^{*}\left(\frac{t-\tau}{a}\right) d t
\end{aligned}
$$

In this equation the parameter ' $a$ ' is a scaling factor which controls the scaling of the signal in frequency and $\tau$ is the shift parameter which controls the signal dilation or contraction in 
time, while $\psi^{*}$ is the function which defines the CWT, known as the mother wavelet from which all daughter wavelets that are used in transformation are derived. ' $*$ ' denotes the complex conjugate.

The mother wavelet is designed such that it can be inverted and the original signal can be retrieved, so that in theory we can write the inverse of the CWT as [27]:

$$
x(t)=\frac{1}{C_{\psi}} \int_{a} \int_{\tau} \frac{1}{a^{2}} x(a, \tau) \psi\left(\frac{t-\tau}{a}\right) d t d a
$$

However, in its continuous form the transform is extremely redundant, involves extensive analytical calculation, and, cannot easily be inverted in practice. To avoid these difficulties, the wavelets are made discrete using scaling and translation parameters $a=2^{-j / 2}$ and $\tau=2^{j} k$ respectively, so that the DWT transformed signal can be written as [27]:

$$
\begin{aligned}
\operatorname{DWTx}(j, k)=\left\langle x, \psi_{j, k}\right\rangle & \\
& =2^{\frac{-j}{2}} \sum_{n=-\infty}^{\infty} x(n) \psi\left(2^{-j} n-k\right)
\end{aligned}
$$

In this study, we have constrained ' $n$ ' into a positive finite region only, since the length of a signal cannot be infinite. This transform can be readily inverted to retrieve the original signal as follows,

$$
x(n)=\sum_{k=0}^{\infty} \sum_{j=-\infty}^{\infty} D W T x(j, k) \psi\left(2^{-j} n-k\right)
$$

With the above baseband architecture, a Multiple Input and Multiple Output (MIMO) front-end is then appended to the DWT-OFDM whereby data is both transmitted and received by multiple antennas. MIMO systems can provide significant increases in data throughput and link reliability without any additional bandwidth usage. This form of baseband and front-end formulation is known to achieve high spectral efficiency and throughput [28]-[30].

\subsection{Proposed Wavelet based Multicarrier System}

The proposed implementation has similarities to FFT-OFDM. On the transmitter side data bits are modulated, for example using BPSK or higher level modulation, mapping the data into symbols, which then pass through a serial to parallel converter to reform as $N$ parallel streams, where $N$ is the number of subchannels. Next, orthogonal wavelet division multiplexing is applied: a pair of data streams $x_{i}(n)$ are up-sampled by a factor of two and then passed through a Quadrature Mirror Filter (QMF) bank that consists of two half-band filters. These are a lowpass filter (LPF), with impulse response $h$, and a highpass filter (HPF) with impulse response $g$. These convolve with the signal, such that $x_{\text {low }}[n]=h[n] * x[n]$ and $x_{\text {high }}[n]=g[n] * x[n]$, where $x[n]$ is the original signal.

The coefficients output from the two filters constitute a wavelet symbol. The combined process of up-conversion and filtering is known as synthesis, and is repeated successively to complete the translation-scaling process. Fig. 1 shows a two level wavelet synthesis process, also called the Inverse Discrete Wavelet Transform (IDWT).

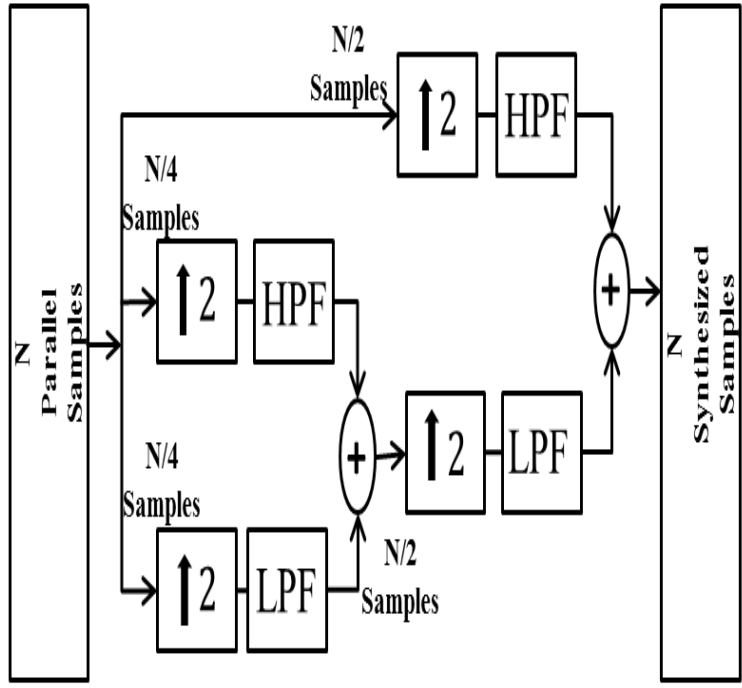

Fig. 1. 2-Level Wavelet Synthesis

The synthesized data is then passed through the channel in the presence of AWGN as shown in Fig. 2.

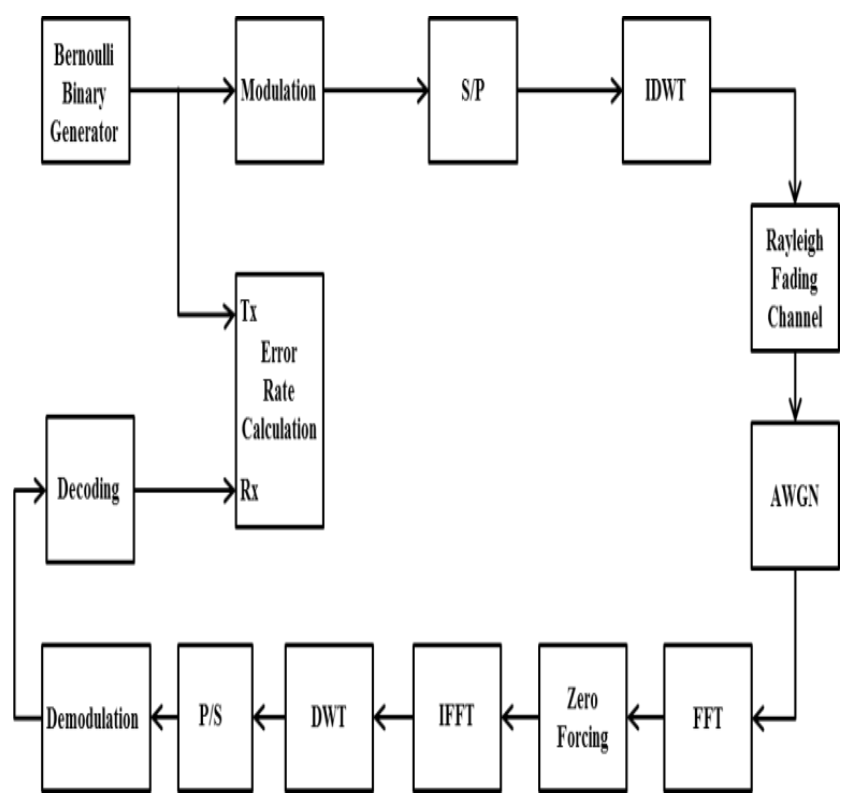

Fig. 2. Proposed DWT multicarrier system.

The convolution between the channel and the signal is linear and the effects are discussed in Part (C).

On the receiver side, the received signal is converted to the frequency domain using FFT and then equalized using zero forcing $(\mathrm{ZF})$ equalization algorithm. Once the received signal is successfully equalized in the frequency domain it is converted back to time domain using IFFT and then analyzed using discrete wavelet transform process. This involves conjugate high pass $h^{*}(-n)$ and low pass filters $g *(-n)$ followed by down-sampling by a factor of 2 . This process is repeated until the initial $N$ parallel streams are obtained, which are passed through a parallel to serial convertor and demodulated. Fig. 3 shows two levels of the DWT. 


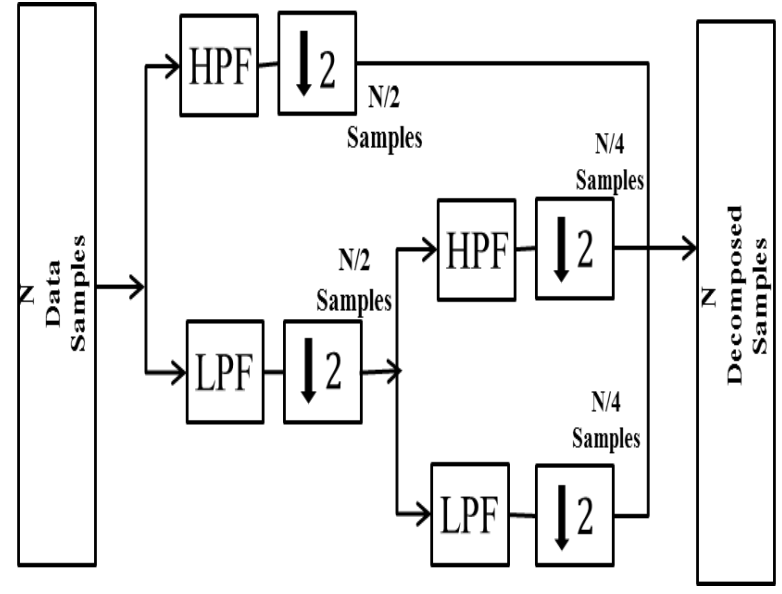

Fig. 3. 2-Level Wavelet Decomposition.

Consider an input sequence, $x(n)$, which is to be transformed by a scaling filter $g(n)$ with coefficients $[\mathrm{g}(0)$, $\mathrm{g}(1), \mathrm{g}(2), \mathrm{g}(3)]$ and wavelet filters $h(n)$ with coefficients $[\mathrm{h}(0), \mathrm{h}(1), \mathrm{h}(2), \mathrm{h}(3)]$. These will be convolved with $x(n)$ according to the following [31], [32];

$\left[\begin{array}{l}p(0) \\ p(1) \\ p(2) \\ p(3) \\ p(4) \\ p(5) \\ \mathrm{M} \\ p(n-1)\end{array}\right]=\left[\begin{array}{cccccccc}h(3) & h(2) & h(1) & h(0) & 0 & \Lambda & 0 & 0 \\ g(3) & g(2) & g(1) & g(0) & 0 & \Lambda & 0 & 0 \\ 0 & 0 & h(3) & h(2) & h(1) & h(0) & \Lambda & 0 \\ 0 & 0 & g(3) & g(2) & g(1) & g(0) & \Lambda & 0 \\ \mathrm{M} & & 0 & & & & \\ h(1) & h(0) & 0 & \Lambda & 0 & 0 & h(3) & h(2) \\ g(1) & g(0) & 0 & \Lambda & 0 & 0 & g(3) & g(2) \\ h(2) & h(1) & h(0) & 0 & \Lambda & 0 & 0 & h(3) \\ g(2) & g(1) & g(0) & 0 & \Lambda & 0 & 0 & g(3)\end{array}\right]\left[\begin{array}{l}x(0) \\ x(1) \\ x(2) \\ x(3) \\ x(4) \\ x(5) \\ \mathrm{M} \\ x(n-1)\end{array}\right]$

By a lifting scheme [32]-[35] the LPF and the HPF can form a single function $\mathrm{p}(\mathrm{n})$, and we can express the relation above in a compact form as;

$$
y(n)=p(n) * x(n)
$$

$p(n)$ in the relation above has been shown in M. Vetterli et al [31] to be a Toeplitz matrix. The shifting by 2 (looking from the left-hand-side) in the matrix equation is produced by the up-sampling (or down-sampling, as the case may be) in the wavelet configuration [32]. The matrix for the filter impulse response must be resized according to the length of the input signal.

As the signal $y(n)$ traverses the multipath channel, the orthogonality of the symbols will be lost but if delays are not longer than the shifts during the scaling and translations, by applying equivalent matching filters, the signals will be correctly reconstructed [36].

The relationship between the high-pass and low-pass filters of QMF banks can be expressed mathematically as:

$$
h(n)=(-1)^{n} g(l+1-n)
$$

Where, $l$ is the length of $g(n)$.

\subsection{Multi-resolution Analysis and Sub- Band Coding}

Various techniques of multi-resolution analysis enable signals to be analyzed into multiple frequency bands. The approach employed in this investigation is called sub-band coding in which signals are divided into several independent sub-bands.

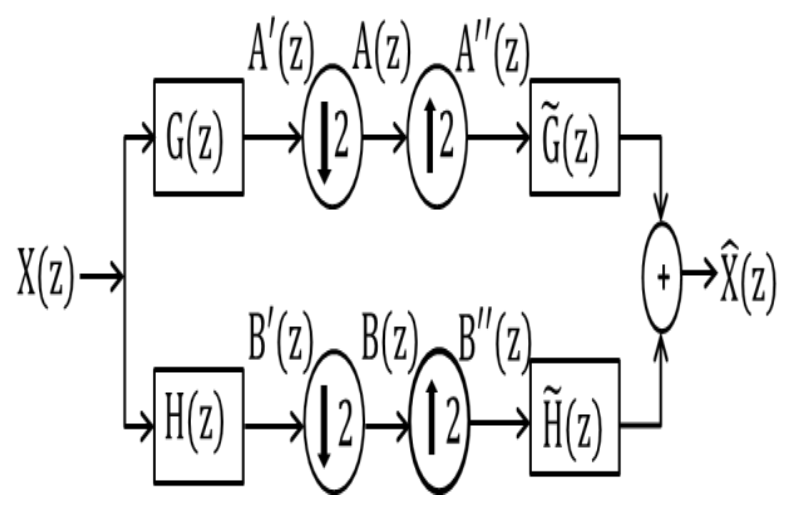

Fig. 4. Two channel sub-band coding analysis and synthesis.

The block diagram in Fig. 4 shows one stage of analysis and synthesis. Two parallel streams are created by de-multiplexing the input signal stream, one of which is analyzed using the low pass filter and the other using the high pass filter.

Meanwhile, the input signal and the filter follow the ztransform tradition as follows;

$$
X(z)=\sum_{n} x(n) z^{-n}
$$

Where,

$$
z^{-n}=e^{-j n \omega}
$$

The intermediate signals $A^{\prime}(z)$ and $B^{\prime}(z)$ that are obtained after the filtering process of $X(z)$ are as follows:

$$
A^{\prime}(z)=X(z) G(z)
$$

And,

$$
B^{\prime}(z)=X(z) H(z)
$$
give:

$A^{`}(z)$ and $B^{`}(z)$ are then down-sampled by a factor of 2 , to

$$
A(z)=\frac{1}{2}\left[A^{\prime}\left(z^{\frac{1}{2}}\right)+A^{\prime}\left(-z^{\frac{1}{2}}\right)\right]
$$

And,

$$
B(z)=\frac{1}{2}\left[B^{\prime}\left(z^{\frac{1}{2}}\right)+B^{\prime}\left(-z^{\frac{1}{2}}\right)\right]
$$

Following the signal path on Fig. 4, these signals are then up-sampled by a factor of 2 to give two intermediate signals as follows:

$$
A^{\prime \prime}(z)=A\left(z^{2}\right)
$$

And,

$$
B^{\prime \prime}(z)=B\left(z^{2}\right)
$$

Now, substituting (10) in (12) and (11) in (13) yields: 


$$
A^{\prime \prime}(z)=\frac{1}{2}\left[A^{\prime}(z)+A^{\prime}(-z)\right]
$$

And,

$$
B^{\prime \prime}(z)=\frac{1}{2}\left[B^{\prime}(z)+B^{\prime}(-z)\right]
$$

Hence the output produced by the filter bank can be expressed by the following:

$$
\hat{X}(z)=A^{\prime \prime}(z) \tilde{G}(z)+B(z) \widetilde{H}(z)
$$

Substituting (16) and (17) into (18), $\hat{X}(z)$ is given by:

$$
\begin{aligned}
\hat{X}(z)=\frac{1}{2}\left[A^{\prime}(z)+\right. & \left.A^{\prime}(-z)\right] \tilde{G}(z) \\
& +\frac{1}{2}\left[B^{\prime}(z)+B^{\prime}(-z)\right] \widetilde{H}(z)
\end{aligned}
$$

It follows that the synthesized output can be given in terms of the original signal, $X(z)$ by substituting the values of $(10)$ and (11) into (19),

$$
\begin{aligned}
\hat{X}(z)=\frac{1}{2}[X(z) & G(z)+X(-z) G(-z)] \tilde{G}(z) \\
+ & \frac{1}{2}[X(z) H(z) \\
+ & X(-z) H(-z)] \widetilde{H}(z)
\end{aligned}
$$

Simplifying (20), results in:

$$
\begin{aligned}
\widehat{X}(z)=\frac{1}{2}[G(z) \widehat{G}(z) & +H(z) \widehat{H}(z)] X(z) \\
& +\frac{1}{2}[G(-z) \hat{G}(z) \\
& +H(-z) \widehat{H}(z)] X(-z)
\end{aligned}
$$

It is worth noting that the estimated signal $\hat{X}(z)$ in (21) contains both the original transmitted signal (first term) and an associated alias part in the second term.

In order to remove the aliasing part, the second term is set to zero, i.e.

$$
G(-z) \hat{G}(z)+H(-z) \widehat{H}(z)=0
$$

To extract a realizable solution for $(20), H(z)$ is taken as a Finite Impulse Response (FIR) filter of order $(N+1)$, where $N$ is always an odd number. Then the following two conditions are applied to compensate the anti-aliasing in (22):

$$
\tilde{G}(z)=-H(z)
$$

And,

$$
G(z)=\widetilde{H}(z)
$$

With anti-aliasing imposed, the output produced by the filter bank $(\hat{X}(z))$, in $(21)$ reduces to:

$$
\hat{X}(z)=\frac{1}{2}[G(z) \tilde{G}(z)+H(z) \widetilde{H}(z)] X(z)
$$

We now need the following well known definitions [30]:

$$
\begin{gathered}
\widetilde{H}(z) \triangleq z^{-N} H\left(z^{-1}\right) \\
\tilde{h}(n) \triangleq h(N-n) \\
\tilde{G}(z) \triangleq z^{-N} G\left(z^{-1}\right)
\end{gathered}
$$

And

$$
\tilde{g}(n) \stackrel{\Delta}{=} g(N-n)
$$

If (23b) and (25a) are combined, $G(z)$ can be expressed in terms of $H\left(z^{-1}\right)$ thus:

$$
G(z)=\widetilde{H}(-z)=-\left(z^{-N}\right) H\left(-z^{-1}\right)
$$

The above equation can also be rewritten in the following format:

$$
G(z)=-z^{-N} H\left(-z^{-1}\right)
$$

Now substituting (23a), (25a) and (28) into (24), $\tilde{X}(z)$ can be written in terms of $H(z)$ as follows:

$$
\begin{array}{r}
\tilde{X}(z)=\frac{1}{2}\left[-z^{-N} H\left(-z^{-1}\right)(-H(-z))\right. \\
\left.+H(z) z^{-N} H\left(z^{-1}\right)\right] X(z)
\end{array}
$$

The simplified form of (29) is given by:

$$
\begin{aligned}
\tilde{X}(z)= & \frac{1}{2}\left[H(-z) H\left(-z^{-1}\right)\right. \\
& \left.+H(z) H\left(z^{-1}\right)\right] z^{-N} X(z)
\end{aligned}
$$

Setting the inner elements within the two brackets of (30), equal to the value 2 :

$$
H(-z) H\left(-z^{-1}\right)+H(z) H\left(-z^{-1}\right)=2
$$

It follows that the estimated signal $\tilde{X}(z)$ will be precisely equal to the transmitted signal $X(z)$ with an $N$ sample delay. If (9), is used in (31), this gives:

$$
\left|H\left(e^{j \omega}\right)\right|^{2}+\left|H\left(e^{j(\omega+\pi)}\right)\right|^{2}=2
$$

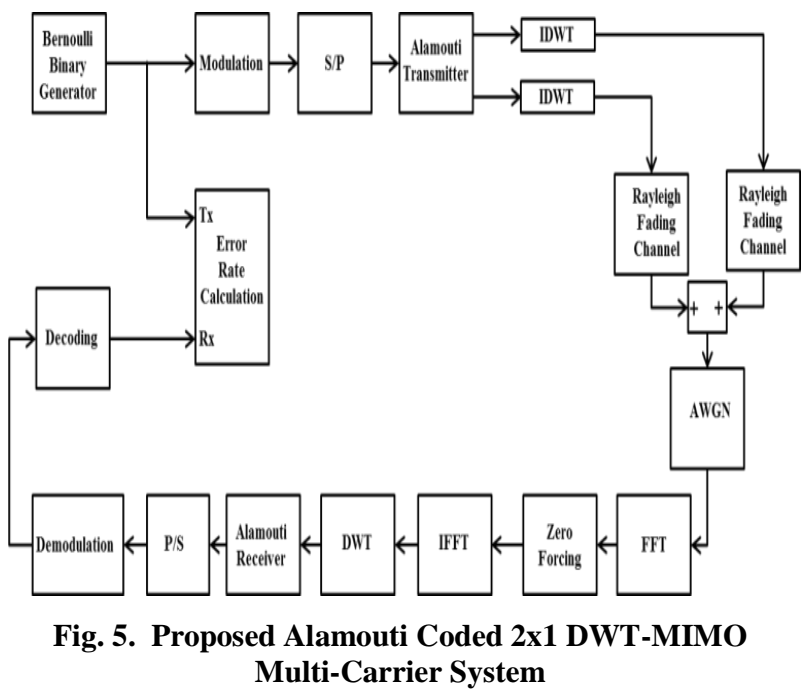

Thus the criterion for perfect reconstruction is expressed by (32) or equivalently by (31). $H(z)$ and $G(z)$ are quadrature mirror filters. If $H(z)$ is known, the three required filters given in (23a), (25a) and (28) can be easily derived to form the perfect reconstruction filter bank.

\subsection{Zero Forcing Equalization}

Channel estimation in wavelet-based multicarrier systems is a challenge, and finite impulse response (FIR) based zeroforcing was proposed in Farrukh et al [22] for a time domain equalization. In this work, we implement frequency-domain equalization. An FFT operation has been performed on the 
wavelet signal at the receiver (see Fig. 2, 5 and 6) according to the following;

$$
S(f) \stackrel{\mathrm{FFT}}{\Longleftrightarrow} X(\tau, a)
$$

Where, $X(\tau, a)$ is the wavelet-domain signal scaled by " $a$ " and shifted by " $\tau$ " and $S(f)$ is the transformed signal which will pass the equalizer.

In conventional OFDM systems where a cyclic prefix is included, linear convolution in the time domain with channel impulse response $h(\tau)$ is equivalent to cyclic convolution. As a result, there is no ISI and frequency domain equalization can be implemented exactly by dividing by the frequency response of the channel, $H$. This is estimated, e.g. by transmitting known training data. If the received signal in the frequency domain is

$$
R=H S+N,
$$

Where, $S$ is the signal spectrum and $N$ is the noise spectrum, then

$$
R / H=S+N / H .
$$

Note that noise will be increased in sub-channels in which $H$ is faded. Where there is no cyclic prefix, then the convolution is not cyclic and the convolution theorem does not apply exactly. Then

$$
R^{\prime}=S+X+N,
$$

Where, $X$ represents the ISI in the frequency domain, introducing an error. It follows that

$$
R^{\prime} / H=S+(X+N) / H \text {. }
$$

In conventional OFDM, symbols transmitted in sub channels where $H$ is faded will experience increased probability of error due to $X$ as well as to $N$. In the proposed system, the ISI is spread by the wavelet transform across a number of wavelet domain symbols. The deleterious effect on error probability is offset by having more symbol power, since none is wasted in transmitting the cyclic prefix.

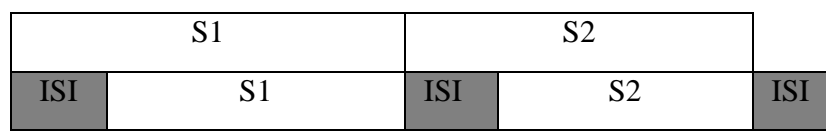

Fig. 6. Production of ISI in the proposed system

\subsection{Maximal Ratio Combining}

The above mentioned technique is now extended to include a receive diversity scheme, using maximal ratio combining to obtain optimal performance, although at the cost of increased complexity. The benefits of using receive diversity, with a number $K$ of different antennas, is to improve the effective $\left(E_{b} / N_{o}\right) K$ times as compared to that of a single receive antenna [32]:

$$
\gamma=\sum_{k=1}^{K} \frac{\left|h_{k}\right|^{2} E_{b}}{N_{o}}
$$

Where, $K$ is the number of the receive antenna, and $\gamma$ is the signal to noise power ratio (SNR) gain as the $K$ increases.

\subsection{Alamouti Coded Wavelet based System}

Next, space-time block coding is introduced, which is also a MIMO technique. Fig. 5 below shows an Alamouti coded wavelet-based OFDM system with two transmitting and one receiving antennas.

At the transmitter the binary data were BPSK modulated and then reformed into parallel data by a serial to parallel converter. After the IDWT process, the data were coded using the Alamouti scheme before transmission over a multipath Rayleigh channel with AWGN. The particular Alamouti code is as follows [37]:

$$
S=\left[\begin{array}{cc}
s_{1} & s_{2}^{*} \\
s_{2} & -s_{1}^{*}
\end{array}\right]
$$

Symbols $s_{1}$ and $s_{2}$ were transmitted in the first time slot $(\mathrm{t})$ over the two channels and $-s^{*}{ }_{1}$ and $-s^{*}{ }_{2}$ (where $*$ again denotes the complex conjugate) were transmitted in the second time slot $(t+T)$. The symbols received can be written as:

$$
R=\left[\begin{array}{ll}
h_{1} & h_{2}
\end{array}\right]\left[\begin{array}{cc}
s_{1} & s_{2}^{*} \\
s_{2} & -s_{1}^{*}
\end{array}\right]
$$

Or, 


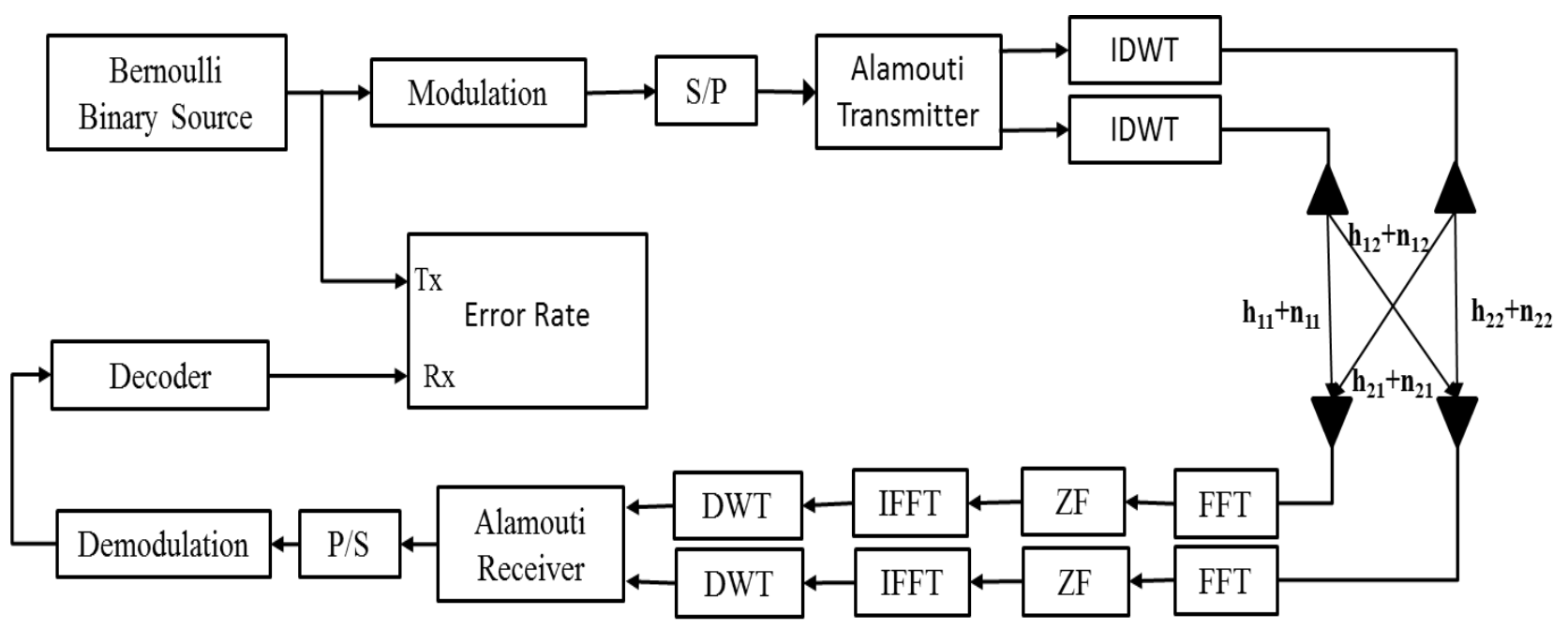

Fig. 7. Proposed Alamouti Coded 2-Transmitter 2-Receiver-based Wavelet Multi Carrier System

$$
R=\left[\begin{array}{ll}
h_{1} s_{1}+h_{2} s_{2} & h_{2} s_{2}^{*}-h_{2} s_{1}^{*}
\end{array}\right]
$$

Where, $h_{k}$ represents the channel. AWGN has been added to each element of $R$.

Now, $R * S^{H}$, where, $(.)^{H}$ stands for the Hermitian transpose, gives:

$$
R S^{H}=h \cdot S \cdot S^{H}=\left[\begin{array}{ll}
h_{1} & h_{2}
\end{array}\right]\left(\left|s_{1}\right|^{2}+\left|s_{2}\right|^{2}\right)
$$

Since,

$$
S . S^{H}=\left(\left|s_{1}\right|^{2}+\left|s_{2}\right|^{2}\right)[I]
$$

Where, $[I]$ is the identity matrix.

Once $R$ is known, for the subsequent unknown symbols, a matrix $Q$ is constructed as:

$$
H=\left[\begin{array}{cc}
h_{1} & h_{2} \\
h_{2}^{*} & -h_{1}^{*}
\end{array}\right]
$$

Then

$$
Q=H s=\left[\begin{array}{c}
R(1) \\
-R(2)^{*}
\end{array}\right]
$$

Therefore

$$
H^{H} Q=\left(\left|h_{1}\right|^{2}+\left|h_{2}\right|^{2}\right)\left[\begin{array}{l}
s_{1} \\
s_{2}
\end{array}\right]
$$

Where, $H H^{H}$ is a diagonal matrix.

Once the symbols are separated they can be decoded in the detector, the serial data passing through the DWT, and the $N$ parallel streams then reformed via the parallel to serial convertor, prior to demodulation.

In Fig. 7 we now show a discrete wavelet transform-based Alamouti-coded two transmitter and two receiver-based system. The transmitter part of the $2 \times 2$ Alamouti coded wavelet-based OFDM is the same as that of $2 \times 1$ but we now have two receive antennas, so that there are four channels involved:

$$
\left[\begin{array}{c}
y_{1}^{1} \\
y_{2}^{1} \\
y_{1}^{2^{*}} \\
y_{2}^{2^{*}}
\end{array}\right]=\left[\begin{array}{cc}
h_{11} & h_{12} \\
h_{21} & h_{22} \\
h_{12}^{*} & -h_{11}^{*} \\
h_{22}^{*} & -h_{21}^{*}
\end{array}\right]\left[\begin{array}{l}
x_{1} \\
x_{2}
\end{array}\right]+\left[\begin{array}{c}
n_{1}^{1} \\
n_{2}^{1} \\
n_{1}^{2^{*}} \\
n_{2}^{2^{*}}
\end{array}\right]
$$

Where, $h_{i j}$ (for $i=1,2$ and $j=1,2$ ) is one of the four independent Rayleigh fading channels. Then after equalization the estimated transmitted symbols can be written as:

$$
\left[\begin{array}{c}
\hat{x}_{1} \\
\hat{x}_{2}^{*}
\end{array}\right]=\left(H H^{H}\right)^{-1} H^{H}\left[\begin{array}{c}
y_{1}^{1} \\
y_{2}^{1} \\
y_{1}^{2^{*}} \\
y_{2}^{2 *}
\end{array}\right]
$$

In this case, equalized serial symbols then pass through three levels of DWT, the parallel to serial convertor and are then demodulated.

\section{SIMULATION AND RESULTS}

In this section the results from the systems described above are discussed. These were obtained through Monte-Carlo simulations. 
The first study was to compare the performances of different wavelet families: not all wavelet families perform alike because they all involve different filters.

The results support the theory that the Daubechies family works best for reconstruction and can provide the best results in multicarrier systems, and Fig. 8 depicts an $\left(E_{b} / N_{o}\right)$ gain of approximately $2 \mathrm{~dB}$ for a BER of $10^{-3}$ in a multipath Rayleigh channel with AWGN.

The simulation was repeated under the same conditions as shown in Table I for Daubechies, Biorthogonal and Reverse Biorthogonal wavelets.

\section{TABLE I}

DWT Simulation PARAMETERS

\begin{tabular}{ll}
\hline \multicolumn{1}{c}{ Parameter } & \multicolumn{1}{c}{ Parameter Value } \\
\hline Modulation scheme & BPSK \\
FFT size & Nil \\
Cyclic prefix & Nil \\
Wavelet families & db8, bior1.3, rbior1.3 \\
Propagation & Rayleigh fading + AWGN \\
Decomposition stages & $\mathrm{K}=\log _{2}(\mathrm{~N}), \mathrm{N}=64$ \\
Symbol length & $2^{6} \times \mathrm{M}, \mathrm{M}=10^{5}$ \\
\hline
\end{tabular}

After selecting the Daubechies wavelet family, a filter length of 8 was found, after several trials, to give the best balance between overall system performance and acceptable processing speed. We next compared the BER performances of the wavelet packet transform-based system and the discrete wavelet-based system. Simulation parameters used for both the DWT and WPT are described in Table II below.

\section{TABLE II}

\section{SimUlation PARAMETERS DWT AND WPT}

\begin{tabular}{|c|c|c|}
\hline Parameter & DWT & WPT \\
\hline $\begin{array}{l}\text { Modulation } \\
\text { scheme }\end{array}$ & 16-QAM, 64-QAM & $\begin{array}{l}\text { 16-QAM, 64- } \\
\text { QAM }\end{array}$ \\
\hline Wavelet family & $\mathrm{db} 8$ & $\mathrm{db} 8$ \\
\hline \multirow{3}{*}{$\begin{array}{l}\text { Propagation } \\
\text { Decomposition } \\
\text { stages } \\
\text { Symbol length }\end{array}$} & $\begin{array}{l}\text { Rayleigh fading + } \\
\text { AWGN }\end{array}$ & $\begin{array}{l}\text { Rayleigh fading + } \\
\text { AWGN }\end{array}$ \\
\hline & $\begin{array}{l}K=\log _{2}(N), N= \\
64\end{array}$ & $\begin{array}{l}\mathrm{K}=\log _{2}(\mathrm{~N}), \mathrm{N}= \\
64\end{array}$ \\
\hline & $2^{6} \times \mathrm{M}, \mathrm{M}=10^{5}$ & $2^{6} \times M, M=10^{5}$ \\
\hline
\end{tabular}

Monte-Carlo simulation results obtained are shown below in Fig. 9.

It was observed through simulations as shown in Fig. 9 that the DWT outperforms the WPT by an $\left(E_{b} / N_{o}\right)$ margin of about $5 \mathrm{~dB}$ for the same BER of $10^{-1}$. The performance of wavelet packet transform based system marginally improves at high $\left(E_{b} / N_{o}\right)$ values, but the discrete wavelet transform still performs better. Thus we selected the DWT for our system.

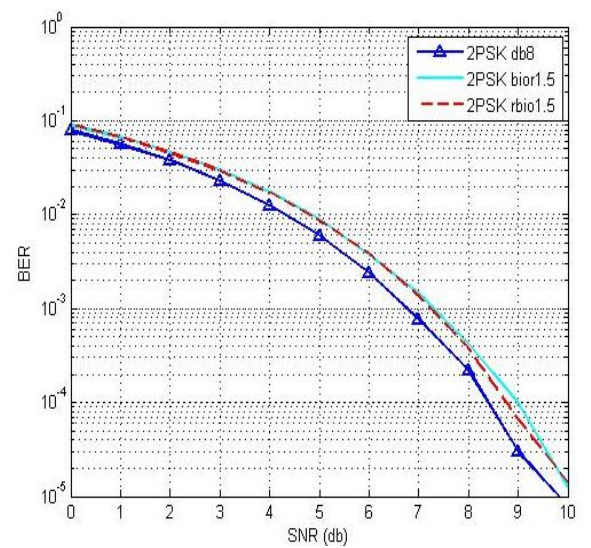

Fig. 8. Performance Comparison of Different Wavelet Families

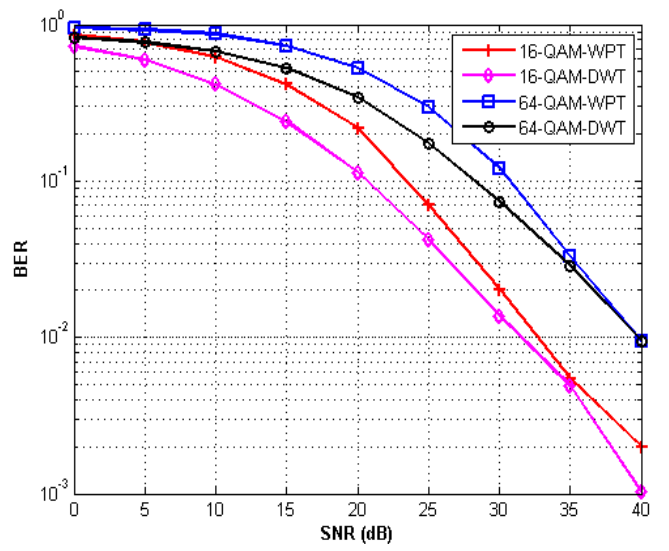

Fig. 9. Performance Comparison OF DWT and WPT in MCS

The final choice is what type of modulation scheme to use Simulations were performed in which we choose four different types of modulations and kept the rest of the simulation parameters the same as in Table I.

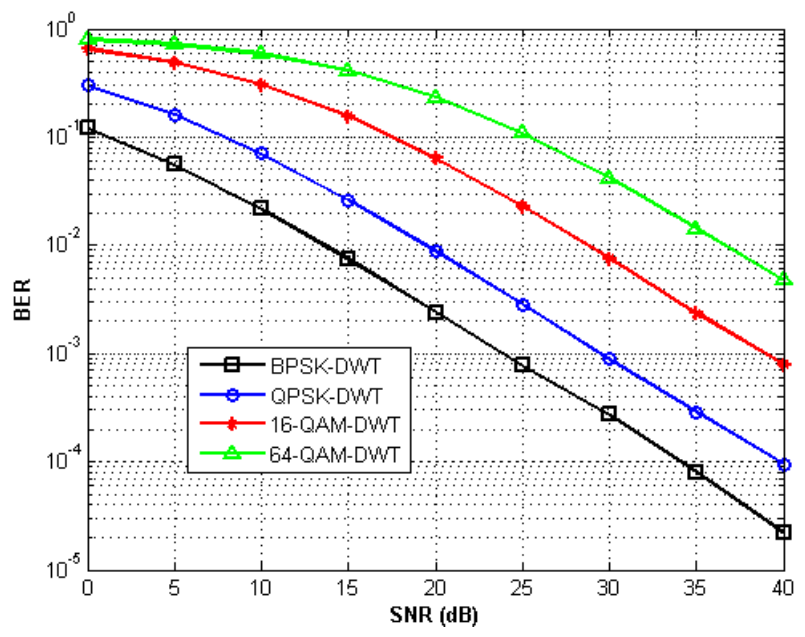

Fig. 10. DWT-based MCS using different modulation schemes

For simplicity and best BER performance BPSK was selected as the modulation scheme for the rest of this study. 
TABLE III

Simulation PaRameters DWT AND FFT

\begin{tabular}{lll}
\hline \hline \multicolumn{1}{c}{ Parameter } & \multicolumn{1}{c}{ DWT } & \multicolumn{1}{c}{ WPT } \\
\hline $\begin{array}{l}\text { Modulation } \\
\text { scheme }\end{array}$ & BPSK & BPSK \\
FFT size & Nil & 64 \\
Wavelet family & db8 & db8 \\
Propagation & Rayleigh fading + & Rayleigh fading + \\
Decomposition & AWGN & AWGN \\
stages & K $=\log _{2}(\mathrm{~N}), \mathrm{N}=$ & $\mathrm{K}=\log _{2}(\mathrm{~N}), \mathrm{N}=$ \\
Symbol length & 64 & 64 \\
& $2^{6} \times \mathrm{M}, \mathrm{M}=10^{5}$ & $2^{6} \times \mathrm{M}, \mathrm{M}=10^{5}$ \\
\hline
\end{tabular}

Next we compared the conventional FFT-OFDM and DWT-based multicarrier systems. The results produced in the Fig. 11 and Fig. 12 clearly suggest that the DWT-based MCS performs better than the conventional Fourier-based OFDM system by a margin in $\left(E_{b} / N_{o}\right)$ of about $6 \mathrm{~dB}$ at a BER of $10^{-4}$ in a multipath fading environment.

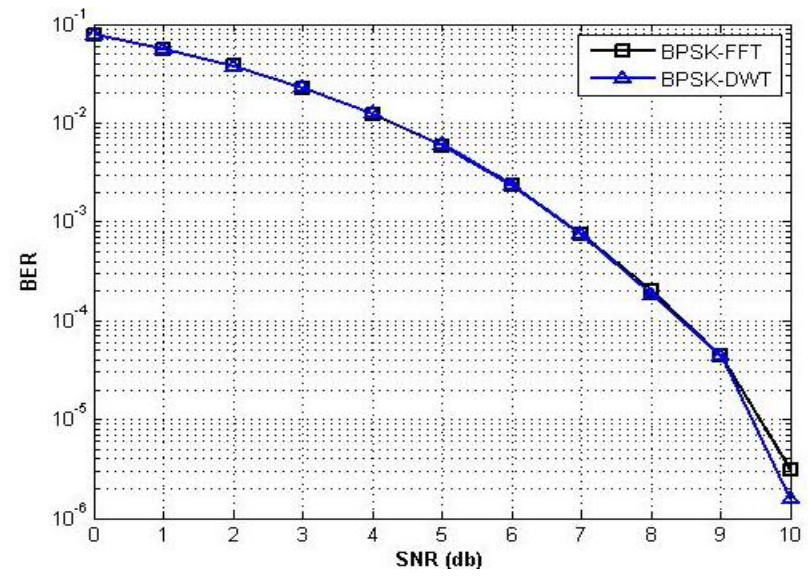

Fig. 11. Comparison of DWT-OFDM and FFT-OFDM in AWGN

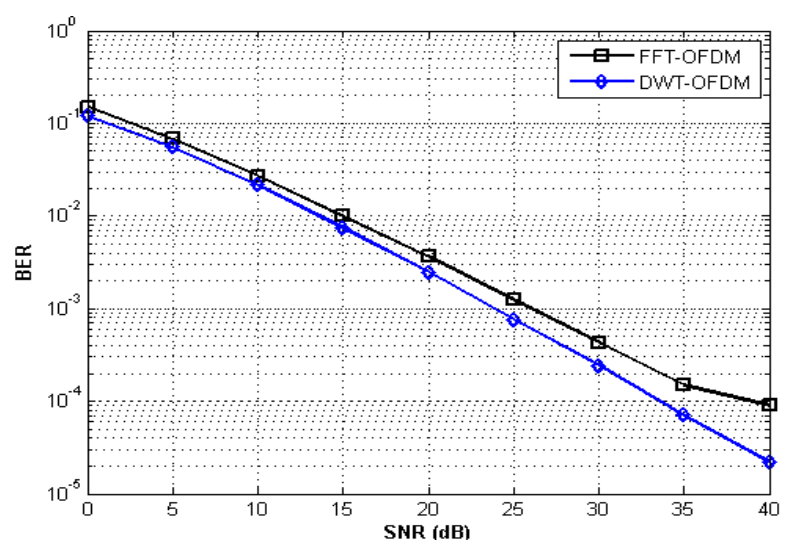

Fig. 12. Performance comparison of DWT-based MCS and FFT-OFDM

The model was then extended to use Maximal Ratio Combining receive diversity.
The results obtained are depicted in Fig. 13 and they show that as the number of antennas on the receiver is increased the performance approaches that of line-of-sight systems. Also, it can be seen that wavelet-based MCS complies with the law of diminishing returns. Thus, the antenna gain decreases with increasing number of antennas.

The Alamouti-based transmit diversity technique was then applied and compared with MRC which showed that two antennas on the transmitter side and one antenna on the receiver side has the same diversity order as that of one transmit and two receive antennas as suggested by Sivash Alamouti [38]. Fig. 14 shows these results.

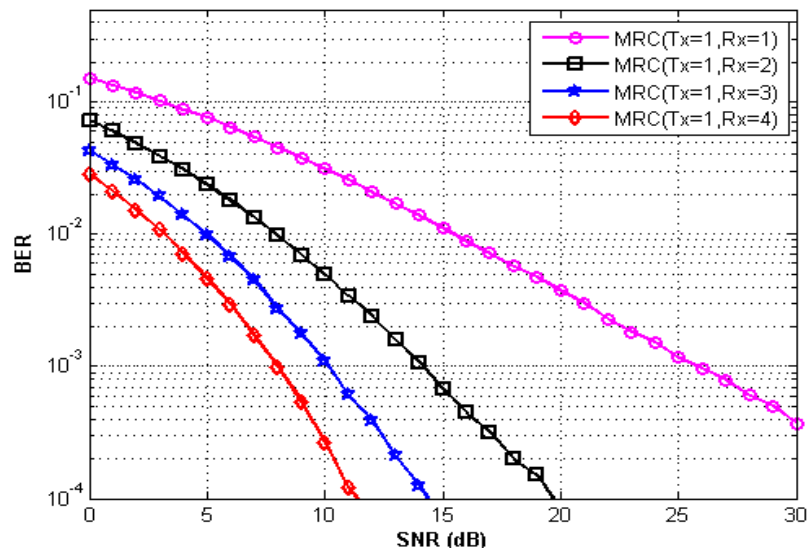

Fig. 13. DWT-MIMO-MCS using Maximal Ratio Combining

\section{CONCLUSION}

In this work, an alternative technique to FFT-OFDM has been presented and investigated, and made comparisons between different wavelet families and different wavelet transforms a frequency-domain channel equalization method using zeroforcing equalization was introduced which showed better performance than conventional FFT-OFDM frequencydomain equalization. The proposed system exploits the special property of wavelet transforms such that filter distortions and signal aliasing is completely cancelled using the analysis and synthesis filters. This resulted in the perfect reconstruction of the original input data signal and perfect extraction of the multiplexed input signals. The aliasing cancellation condition that is imposed in the filter banks ensures no cross talk in the corresponding transmultiplexer. Meanwhile, the proposed wavelet transform multicarrier modulation scheme brought increased spectral efficiency, consequent on the non-inclusion of cyclic prefixing which needs up to $25 \%$ of the transmit bandwidth in the conventional FFT-based OFDM. Comparison between two different types of wavelet-based modulations was also presented which showed that the DWT performs better than WPT both in terms of BER performance, and in terms of processing times as WPT decomposition is performed at each node. Diversity techniques were then studied using the proposed DWT-OFDM with frequency domain equalization. Overall discrete wavelet transformbased MC system performed better than the conventional OFDM system in terms of BER performance with savings of $8 \%$ to $25 \%$ bandwidth.

\section{REFERENCES}

[1] P. H. Young, Electronic Communications Techniques, Prentice Hall, Englewood Cliffs, NJ, 1994.

[2] J. G. Proakis, Digital Communications, McGraw-Hill, 1995. 
[3] J. G. Proakis, M. Salehi, and G. Bauch, Contemporary communication systems using MATLAB, Brooks/Cole, 2000.

[4] E. Telatar, "Capacity of Multiple antenna Gaussian Channels," European transactions on telecommunications, vol. 10, no. 6, pp. 585-595, 1999.

[5] Z. Hong, et al., "Spatial multiplexing in correlated fading via the virtual channel representation," IEEE Journal on Selected Areas in Communications, vol. 21, no. 5, pp. 856-866, 2003.

[6] S. Hara, and R. Prasad, Multicarrier techniques for $4 \mathrm{G}$ mobile communications, Artech House Publishers, 2003.

[7] K. Abdullah, N. Al-Hinai, A. Z. Sadik and Z. M. Hussain, "Circular 16-QAM modulation scheme for wavelet and Fourier based OFDM systems," $5^{\text {th }}$ IEEE GCC Conference and Exhibition, pp. 1 - 5, 2009.

[8] V. Kumbasarand O. Kucur, "Better wavelet packet tree structures for PAPR reduction in WOFDM systems," Digital Signal Processing, vol. 18, no. 6, pp. 885-891, 2008.

[9] B. G. Negash, H. Nikookar, "Wavelet based OFDM for wireless channels," Vehicular Technology Conference, IEEE VTS 53 ${ }^{\text {rd }}$, vol. 1, pp. 688-691, 2001.

[10] I. Widad, "Implementation of WiMAX (IEEE802. 16. d) OFDM Baseband Transceiver-Based Multi wavelet OFDM on a Multi-Core Software-Defined Radio Platform," ISRN Signal Processing, 2011.

[11] H. J. Taha, M. F. M. Salleh, "Performance analysis of QAM modulation parameters on wavelet packet transform (WPT) and FFT- OFDM system," IEEE 9th Malaysia International Conference on Communications (MICC), pp. 1-5, 2009.

[12] M. Gautier, M. Arndt, J. Lienard, "Efficient Wavelet Packet Modulation for Wireless Communication," The Third Advanced International Conference on Telecommunications (AICT), pp. 19 - 24, 2007.

[13] B. G. Negash and H. Nikookar, "Wavelet-based multicarrier transmission over multipath wireless channels," Electronics Letters, vol. 36, no. 21, pp. 17871788,2000

[14] M. Gautier, C. Lereau, M. Arndt, J. Lienard, "PAPR analysis in wavelet packet modulation," $3^{\text {rd }}$ International Symposium on Communications, Control and Signal Processing (ISCCSP), pp 799-803, 2008.

[15] L. Zbydniewski, T. Zielinski, “OFDM vs. Wavelet OFDM and Circular Wavelet-OFDM in high speed communication over power lines," Signal Processing Algorithms, Architectures, Arrangements and Applications Conference Proceedings (SPA), pp. 76 - 81, 2009.

[16] A. K. Jain, Fundamentals of Digital Image Processing, Prentice-Hall of

India, New Delhi, 1995.

[17] C. S. Burrus, R. A. Gopinath and H. Guo, "Introduction to Wavelets and Wavelet Transforms-A Primer, Prentice-Hall, New Jersey, USA, 1998.

[18] U. Khan, S. Baig and M. J. Mughal, "Performance Comparison of Wavelet Packet Modulation and OFDM over Multipath Wireless Channel with Narrowband Interference," International Journal of Electrical Engineering and Computer Sciences, IJECS, Spet. 2009 vol.9, no.9, pp.431-434.

[19] V. Kumbasar, et al., "Optimization of wavelet based OFDM for multipath powerline channel by genetic algorithm", Wireless Communications and Mobile Computing, vol. 9, no. 9, pp. 1243-1250, 2009.

[20] G. Strang and T. Nguyen, Wavelets and filter banks, Cambridge University Press, 1996.

[21] M. K. Gupta, S. Shrivastava, A. S. Raghuvanshi, S. Tiwari, "Channel Estimation for Wavelet Based OFDM Systems," International Conference on Devices and Communications (ICDeCom), pp. 1 - 4, 2011.

[22] F. Farrukh, S. Baig, M. J. Mughal, "Performance Comparison of DFT-OFDM and Wavelet-OFDM with Zero Forcing Equalizer for FIR Channel Equalization," International Conference on Electrical Engineering (ICEE), pp 1-5, 2007.

[23] A. Jaminand P. Mahonen, "Wavelet packet modulation for wireless communications," Wireless Communications and Mobile Computing, vol. 5, no. 2, pp. 123-137, March 2005.

[24] T. Nawaz and S. Baig, "Wavelet OFDM- A solution for reliable communication in a frequency selective Rayleigh fading channel," 9th International Bhurban Conference on Applied Sciences and Technology (IBCAST), pp. 413 - 417, 2012.

[25] R. Asif, R. A. Abd-alhameed, O. O. Anoh, Y. A. S. Dama, "Performance Evaluation of DWT-OFDM and FFT-OFDM for Multicarrier Communications Systems using Time Domain Zero Forcing Equalization,' International Journal of Computer Applications, vol. 51- no. 4, pp. 34-38, August 2012.

[26] J. Sadowsky, "Investigation of signal characteristics using the continuous wavelet transform," Johns Hopkins applied technical digest, vol. 17, no. 3, pp. 258-269, 1996.

[27] I. Daubechies, Ten lectures on wavelets, Society for Industrial Mathematics, vol. 61, 1992.

[28] N. M. Shafik, "Wavelet Transform Effect on MIMOOFDM System Performance," Cyber Journals: Multidisciplinary Journals in Science and Technology, Journal of Selected Areas in Telecommunications (JSAT), pp. 46 - 50, September Edition, 2011.

[29] D. Gesbert, et al., "From theory to practice: an overview of MIMO space-time coded wireless systems," IEEE Journal on Selected Areas in Communications, vol. 21, no. 2, pp. 281-302.

[30] S. L. Linfoot, M.K. Ibrahim, and M.M. Al-Akaidi, "Orthogonal wavelet division multiplex: an alternative to OFDM," IEEE Transactions on Consumer Electronics, vol. 53, no. 2, pp. 278-284, May 2007.

[31] M. Vetterli and C. Herley, "Wavelets and Filter Banks: Theory and Design," IEEE Transaction on Signal Processing, vol.40, no.9, pp.2207 - 2231, Sept. 1992,

[32] M. Weeks, Digital Signal Processing Using MATLAB and Wavelets, Second Edition, Jones and Bartlett Publishers, London, 2011. 
[33] W. Sweldens, "The Lifting Scheme: A New Philosophy in Biorthogonal Wavelet Constructions," Wavelet Applications in Signal and Image Processing III, vol.2569, pp.68 - 79

[34] W. Sweldens, "The Lifting Scheme: A Custom-Design Construction of Biorthogonal Wavelets," Applied and Computational Harmonic Analysis, vol.3, no. 0015, pp.186 - 200, 1996.

[35] W. Sweldens, "The Lifting Scheme: A Construction of Second Generation Wavelets," SIAM Journal on Mathematical Analysis, vol. 29, no. 2, pp.511-546, 1997.

[36] D. Daly, C. Heneghan, A. Fagan and M. Vetterli, "Optimal Wavelet Packet Modulation under Finite
Complexity Constraint," IEEE International Conference on Acoustics, Speech, and Signal Processing (ICASSP), pp. III-2789 - III-2792, May 2002.

[37] S. Gracias, V. U. Reddy, "An equalization algorithm for wavelet packet based modulation schemes," IEEE Transactions on Signal Processing, vol. 46, no. 11, pp. 3082-3087, 1998.

[38] S. M. Alamouti, "A simple Transmit Diversity Technique for wireless communications," IEEE Journal on Selected Areas in Communications, vol. 16, no. 8, pp. $1451-1458,1998$. 